\title{
A Case of Sporadic Suprasellar Hemangioblastoma Mimicking Meningioma
}

\author{
Byung-moo Kang, Sang Min Youn \\ Department of Neurosurgery, Korea Cancer Center Hospital, Korea Institute of Radiological and Medical Sciences, Seoul, Korea
}

\author{
Received July 28, 2019 \\ Revised August 19, 2019 \\ Accepted August 27, 2019 \\ Correspondence \\ Sang Min Youn \\ Department of Neurosurgery, \\ Korea Cancer Center Hospital, \\ Korea Institute of Radiological \\ and Medical Sciences, \\ 75 Nowon-ro, Nowon-gu, \\ Seoul 01812, Korea \\ Tel: +82-2-970-1282 \\ Fax: +82-2-970-1967 \\ E-mail: smyoun@kirams.re.kr
}

\begin{abstract}
Hemangioblastoma (HBL) in the suprasellar region is very rare and a few cases have been reported. Suprasellar HBL without von Hippel-Lindau disease is much rarer. A 76-year old male patient presented progressively deteriorating visual disturbance. MRI demonstrated solid suprasellar mass of $20 \mathrm{~mm}$ in diameter, broadly based to planum sphenoidale and diaphragm sella and dural tail sign after the administration of gadolinium diethylene triamine penta-acetic acid (Gd-DTPA). Preoperative diagnosis was meningioma. Total resection of the tumor was not accomplished because of massive hemorrhage, and the histopathologic examination revealed the tumor to be HBL. The visual disturbance of the patient was not improved. The authors reviewed the literature and considered a differential diagnosis of suprasellar tumors and treatment of suprasellar HBL.
\end{abstract}

Key Words $\quad$ Hemangioblastoma; Suprasellar; von Hippel Lindau disease.

\section{INTRODUCTION}

Hemangioblastomas (HBLs) are World Health Organization grade I central nervous system neoplasms and occur sporadically ( $60 \%$ to $75 \%$ of cases) or in the autosomal dominant neoplasia syndrome, von Hippel-Lindau (VHL) disease [1,2]. HBLs represent about $2 \%$ of all intracranial neoplasms and $7 \%$ to $12 \%$ of posterior fossa tumors [3,4]. Over $95 \%$ of HBLs are found in the brain stem, spinal cord, and cerebellum, $1 \%$ to $5 \%$ of HBLs are found in the supratentorial compartment and $10 \%$ of patients with VHL disease have supratentorial HBLs $[5,6]$. When they are found supratentorially, they most frequently (30\%) originate in the region of the pituitary stalk and tuber cinereum [7]. The authors experienced a case of suprasellar HBL mimicking meningioma and reviewed the literature.

This is an Open Access article distributed under the terms of the Creative Commons Attribution Non-Commercial License (https://creativecommons.org/licenses/by-nc/4.0) which permits unrestricted non-commercial use, distribution, and reproduction in any medium, provided the original work is properly cited.

Copyright $\odot 2019$ The Korean Brain Tumor Society, The Korean Society for NeuroOncology, and The Korean Society for Pediatric Neuro-Oncology

\section{CASE REPORT}

A 76-year-old male patient presented with decreased visual acuity and visual field defect. The patient had past medical history of prostate cancer with lung metastasis for 6 years and was under hormonal therapy with cyproterone acetate. He also had deep vein thrombosis and had been taking warfarin. Ophthalmologic examination revealed that visual acuity of his left eye was 0.8 and color defect was found by Ishihara color test. Superior temporal quadrantanopsia of the left eye was also found. MRI demonstrated a solid suprasellar mass of $20 \mathrm{~mm}$ in diameter, broadly based to planum sphenoidale and diaphragm sella. The mass was isointense on T1-weighted images and hyperintense on T2-weighted images. Optic chiasm compression and posterior displacement of pituitary stalk were also shown. Strong enhancement and dural tail sign was observed after the administration of gadolinium diethylene triamine penta-acetic acid (Gd-DTPA) (Fig. 1). The presumptive diagnosis was meningioma. Cerebral angiography was not performed. On consideration of his medical condition, surgical removal was postponed. Six months later, visual disturbance of his left eye was deteriorated and he could perceive only hand movement. Follow up MRI did not demonstrate any change in size of the 
tumor.

Operative resection of the tumor was performed with right frontotemporal craniotomy. In the surgical field, the tumor was round and dark-reddish color, encapsulated with thin wall, broadly based to planum sphenoidale and diaphragm sella. The tumor was removed by piecemeal fashion, massive bleeding occurred during the operative procedure. Total resection of tumor was not accomplished due to hemorrhage. The visual disturbance of the patient was not improved postoperatively.

On histopathologic examination, different two components were seen in low magnification of hematoxylin and eosin staining. Vascular structure consisted with variable sized capillary vessels with thin wall were closely packed, and stromal cells with vacuolated cytoplasm and hyperchromatic nuclei were seen between the vascular structure. Vascular structure cells showed strong immunoreactivity for CD34, whereas stromal cells were negative. Stromal cells were also negative for glial fibrillary acidic protein staining and expressed diffuse positivity with S-100 and neuron specific enolase staining (Fig. 2). These findings are consistent with HBLs.

The authors recommended radiosurgery using CyberKnife (Accuray Incorporated, Sunnyvale, CA, USA) after the remission of postoperative delirium, however, the patient refused adjuvant treatment because the prostate cancer of the patient was progressing in spite of hormonal therapy and there were no treatment plan of the prostate cancer.

After the histopathologic diagnosis, medical chart and im- aging studies previously taken were re-evaluated to reveal the existence of VHL disease. The patient had no family history of VHL disease and did not meet the criteria of VHL disease in spite of simple cyst in his kidney.

The institutional review board exempted informed consent due to its retrospective nature and minimal risk for harm to the patient, and this report was conducted according to the guidelines of the Declaration of Helsinki for biomedical research.

\section{DISCUSSION}

HBLs are benign, vascular tumors of uncertain origin principally composed of stromal and endothelial cell components and comprise $1.1 \%$ to $2.4 \%$ of all intracranial space occupying lesions, occur sporadically or in VHL disease [8]. HBLs usually arise in brain stem, spinal cord, and cerebellum. One percent to $5 \%$ of HBLs are found in the supratentorial compartment $[5,6]$. However, sporadic supratentorial HBLs are very rarely reported in the literature. Mills et al. [8] reported in their review article that $60 \%$ of supratentorial HBL (59 out of 99 ) received a diagnosis of VHL disease. In addition, tumors located in the sellar and suprasellar region are even more likely to be associated with VHL disease [8]. The most frequent symptoms of the patients with suprasellar HBLs are visual and hormonal disturbances [9]. The authors summarized the features of 8 cases of sporadic suprasellar HBLs (including pituitary stalk HBLs) previously reported along with our case in Table 1.
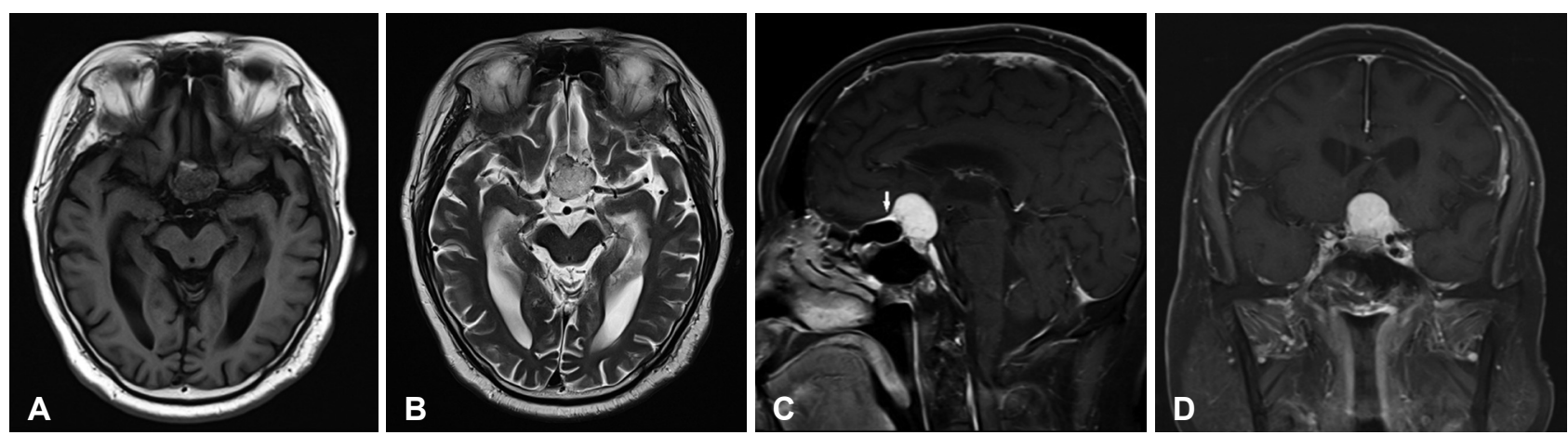

Fig. 1. Preoperative axial MRI without contrast enhancement showing isointense on T1-weighted image and hyperintense on T2-weighted image suprasellar mass of $20 \mathrm{~mm}$ in diameter (A and B). Sagittal and coronal image with contrast enhancement demonstrated homogeneously enhancing solid mass broadly based to planum sphenoidale and diaphragm sella and strong dural tail sign (arrow) (C and D).
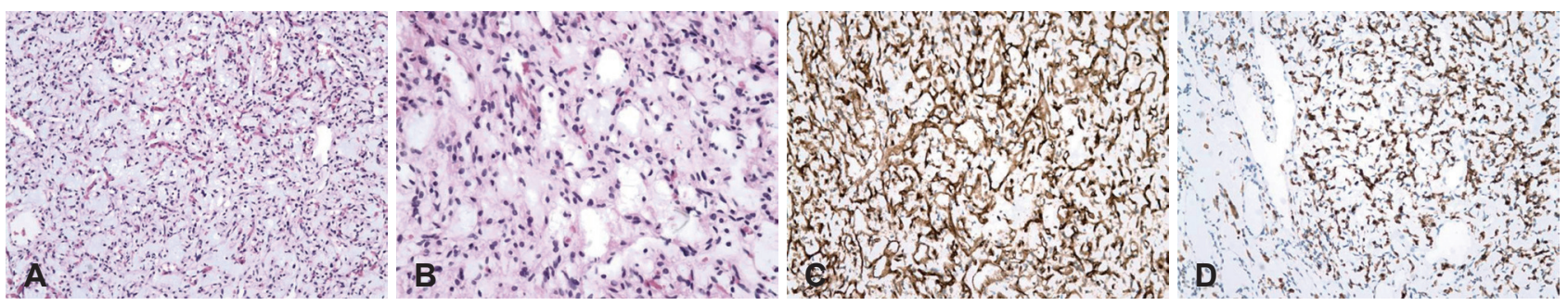

Fig. 2. Hematoxylin and eosin staining showing two different components characterized by numerous thin walled capillary vessels and stromal cells, which are characteristically clear and vacuolated cytoplasm were seen in low and high magnification $[\times 200(A), \times 400(B)]$. Immunostaining for CD34 highlighted the rich vascular network (C) and stromal cells showed immunoreactivity for neuron specific enolase (D). 
Table 1. Literature review of suprasellar hemangioblastoma without von Hippel-Lindau disease

\begin{tabular}{|c|c|c|c|c|c|}
\hline Study & Age/sex & Symptoms & Location & Surgery & Follow-up \\
\hline Grisoli et al. [15] & $28 / \mathrm{F}$ & Headache, galactorrhea, alopecia & Pituitary stalk & TR & Panhypopituitarism \\
\hline Neumann et al. [4] & $35 / \mathrm{F}$ & Headache, amenorrhea, diabetes & Pituitary stalk & N/A & N/A \\
\hline Ikeda et al. [16] & $62 / \mathrm{M}$ & Visual disturbance & Suprasellar & TR & N/A \\
\hline Rumboldt et al. [17] & $60 / \mathrm{M}$ & Visual disturbance, panhypopituitarism & Sellar; suprasellar & TSA biopsy & N/A \\
\hline Pecker et al. [10] & $54 / \mathrm{M}$ & Visual loss & Suprasellar & TR & No recurrence 5 years \\
\hline Xie et al. [14] & $64 / \mathrm{F}$ & Headache, visual disturbance & Suprasellar & STR & CSF leakage, hydrocephalus \\
\hline Lee et al. [12] & $60 / \mathrm{F}$ & Headache, diplopia & Pituitary stalk & TR & Transient DI \\
\hline Li et al. [11] & $51 / \mathrm{F}$ & $\begin{array}{l}\text { Headache, visual disturbance, } \\
\text { hypocortisolism }\end{array}$ & Suprasellar & TR & No recurrence 1 year \\
\hline Our case & $76 / \mathrm{M}$ & Visual disturbance & Suprasellar & STR & No improvement \\
\hline
\end{tabular}

F, female; M, male; TR, total resection; STR, subtotal resection; TSA, trans-sphenoidal approach; CSF, cerebrospinal fluid; DI, diabetes insipidus; N/A, not available

Suprasellar HBLs may be misdiagnosed preoperatively as meningioma, pituitary tumor or craniopharyngioma. The characteristic dural tail sign is usually absent in HBLs [10]. HBLs enhance more strongly with contrast than pituitary tumor and contrast-enhanced coronal T1-weighted images generally identify the diaphragm sella between the tumor and pituitary gland in suprasellar HBLs [10,11]. Lee et al. [12] reported a case of sporadic HBL in the pituitary stalk and reviewed the literature that among 14 cases of HBLs in the pituitary stalk, only 3 cases are not associated with VHL disease. However, they were able to discriminate HBLs and other tumorous condition preoperatively because the mass was beyond the diaphragm sella and absent dural tail sign, a characteristic finding of meningioma [12]. The onset of the disease is usually a differential point with craniopharyngioma. Sawin et al. [13] reported a case of intrasellar HBL occurring in an 11-year-old child. In spite of the family history of VHL disease, craniopharyngioma would be the most probable diagnostic consideration for an intrasellar tumor in a child of this age statistically. Xie et al. [14] insisted that three dimensional fast-imaging employing steady acquisition sequence magnetic resonance (3D-FIESTA MR), three dimensional magnetization prepared rapid acquisition with gradient echo sequence magnetic resonance (3D-MPRAGE MR) and computed tomography angiography (CTA) supplied much more information. The cyst in the tumor must be the blood sinus formed by hemangioblastoma by 3D-MPRAGE $\mathrm{MR}$, and flow voids around the tumor were more clearly on 3D-FIESTA MR. CTA revealed that the mass was hypervascular, supplied by multiple small blood vessels [14].

In our case, preoperative diagnosis was meningioma. The patient had no family history and stigmata of VHL disease. On MR images, faint signal void was demonstrated within the tumor, which was broadly based to planum sphenoidale and diaphragm sella. The tumor also demonstrated strong dural tail sign after the administration of Gd-DTPA (Fig. 1C, arrow).
Gross total resection (GTR) of tumor alone seems to provide excellent tumor control rates. Of the 79 cases that reported extent of resection, 61 (77\%) achieved GTR, 15 achieved subtotal resection (STR). The patient undergoing GTR experienced a significant improvement in progression-free survival (PFS) compared those receiving STR (5-year PFS: GTR 100\% vs. STR $53 \%$; log rank, $p<0.01)$ In addition, patients undergoing GRT experienced a significant improvement in PFS compared with those receiving fractionated radiotherapy (RT) alone (5-year PFS: GTR $100 \%$ vs. RT $33 \%$; log rank, $p<0.001$ ) [8].

The author experienced a rare case of suprasellar HBL without VHL disease mimicking meningioma. HBL should be included in the differential diagnosis of suprasellar tumor preoperatively and GTR of tumor should be tried to achieve excellent tumor control.

\section{Conflicts of Interest}

The authors have no potential conflicts of interest.

\section{REFERENCES}

1. Browne TR, Adams RD, Roberson GH. Hemangioblastoma of the spinal cord. Review and report of five cases. Arch Neurol 1976;33:435-41.

2. Conway JE, Chou D, Clatterbuck RE, Brem H, Long DM, Rigamonti D. Hemangioblastomas of the central nervous system in von HippelLindau syndrome and sporadic disease. Neurosurgery 2001;48:55-62; discussion 62-3.

3. Constans JP, Meder F, Maiuri F, Donzelli R, Spaziante R, de Divitiis E. Posterior fossa hemangioblastomas. Surg Neurol 1986;25:269-75.

4. Neumann HP, Eggert HR, Weigel K, Friedburg H, Wiestler OD, Schollmeyer P. Hemangioblastomas of the central nervous system. A 10year study with special reference to von Hippel-Lindau syndrome. J Neurosurg 1989;70:24-30.

5. Wanebo JE, Lonser RR, Glenn GM, Oldfield EH. The natural history of hemangioblastomas of the central nervous system in patients with von Hippel-Lindau disease. J Neurosurg 2003;98:82-94.

6. Lonser RR, Butman JA, Huntoon K, et al. Prospective natural history study of central nervous system hemangioblastomas in von HippelLindau disease. J Neurosurg 2014;120:1055-62.

7. Lonser RR, Butman JA, Kiringoda R, Song D, Oldfield EH. Pituitary stalk hemangioblastomas in von Hippel-Lindau disease. J Neurosurg 
2009;110:350-3.

8. Mills SA, Oh MC, Rutkowski MJ, Sughrue ME, Barani IJ, Parsa AT. Supratentorial hemangioblastoma: clinical features, prognosis, and predictive value of location for von Hippel-Lindau disease. Neuro Oncol 2012;14:1097-104.

9. Miyata S, Mikami T, Minamida Y, Akiyama Y, Houkin K. Suprasellar hemangioblastoma. J Neuro-Ophthalmol 2008;28:325-6.

10. Peker S, Kurtkaya-Yapicier O, Sun I, Sav A, Pamir MN. Suprasellar haemangioblastoma. Report of two cases and review of the literature. J Clin Neurosci 2005;12:85-9.

11. Li Z, Feng T, Teng H, Hu Y, Yao Y, Liu Y. Suprasellar hemangioblastoma without von Hippel-Lindau disease: a case report and literature review. Int J Clin Exp Pathol 2015;8:7553-8.

12. Lee GI, Kim JM, Choi KS, Kim CH. Sporadic hemangioblastoma in the pituitary stalk: a case report and review of the literature. J Korean
Neurosurg Soc 2015;57:465-8

13. Sawin PD, Follett KA, Wen BC, Laws ER Jr. Symptomatic intrasellar hemangioblastoma in a child treated with subtotal resection and adjuvant radiosurgery. J Neurosurg 1996;84:1046-50.

14. Xie T, Zhang X, Hu F, et al. Suprasellar hemangioblastoma mimicking a craniopharyngioma: result of extended endoscopic transsphenoidal approach--case report. Neurol Med Chir (Tokyo) 2013;53:735-9.

15. Grisoli F, Gambarelli D, Raybaud C, Guibout M, Leclercq T. Suprasellar hemangioblastoma. Surg Neurol 1984; 22:257-62.

16. Ikeda M, Asada M, Yamashita H, Ishikawa A, Tamaki N. [A case of suprasellar hemangioblastoma with thoracic meningioma]. No Shinkei Geka 2001;29:679-83.

17. Rumboldt Z, Gnjidić Z, Talan-Hranilović J, Vrkljan M. Intrasellar hemangioblastoma: characteristic prominent vessels on MR imaging. AJR Am J Roentgenol 2003;180:1480-1. 